\title{
Endoplasmic Reticulum Stress Modulation as a Target for Ameliorating Effects of Blast Induced Traumatic Brain Injury
}

\author{
Brandon P. Lucke-Wold, ${ }^{1, *}$ Aric F. Logsdon, ${ }^{2, *}$ Ryan C. Turner, \\ Jason D. Huber, and Charles L. Rosen ${ }^{1}$
}

\begin{abstract}
Blast traumatic brain injury (bTBI) has been shown to contribute to progressive neurodegenerative disease. Recent evidence suggests that endoplasmic reticulum (ER) stress is a mechanistic link between acute neurotrauma and progressive tauopathy. We propose that ER stress contributes to extensive behavioral changes associated with a chronic traumatic encephalopathy (CTE)-like phenotype. Targeting ER stress is a promising option for the treatment of neurotrauma-related neurodegeneration, which warrants investigation. Utilizing our validated and clinically relevant Sprague-Dawley blast model, we investigated a time course of mechanistic changes that occur following bTBI (50 psi) including: ER stress activation, iron-mediated toxicity, and tauopathy via Western blot and immunohistochemistry. These changes were associated with behavioral alterations measured by the Elevated Plus Maze (EPM), Forced Swim Test (FST), and Morris Water Maze (MWM). Following characterization, salubrinal, an ER stress modulator, was given at a concentration of $1 \mathrm{mg} / \mathrm{kg}$ post-blast, and its mechanism of action was determined in vitro. bTBI significantly increased markers of injury in the cortex of the left hemisphere: p-PERK and p-eIF $2 \alpha$ at $30 \mathrm{~min}, \mathrm{p}$-T205 tau at $6 \mathrm{~h}$, and iron at $24 \mathrm{~h}$. bTBI animals spent more time immobile on the FST at $72 \mathrm{~h}$ and more time in the open arm of the EPM at 7 days. Further, bTBI caused a significant learning disruption measured with MWM at 21 days post-blast, with persistent tau changes. Salubrinal successfully reduced ER stress markers in vivo and in vitro while significantly improving performance on the EPM. bTBI causes robust biochemical changes that contribute to neurodegeneration, but these changes may be targeted with ER stress modulators.
\end{abstract}

Keywords: bTBI; ER stress; iron toxicity, salubrinal; tauopathy

\section{Introduction}

B LAST EXPOSURE has been linked to progressive neurodegenerative changes. Several former war fighters have been diagnosed with neurodegenerative tauopathy and chronic traumatic encephalopathy (CTE) on postmortem examination. ${ }^{1}$ Shively and colleagues found that war fighters exposed to blast had extensive gliosis at the gray/white junction and vessel/brain interfaces. ${ }^{2}$ The injury associated with blast exposure is significantly different than that obtained from rotational brain injury alone. ${ }^{3}$ Blast exposure causes a unique pressure spike that can disrupt the blood-brain barrier $(\mathrm{BBB})$ and contribute to the activation of secondary injury cascades. ${ }^{4}$ What is unknown, is how these secondary injury cascades contribute to the pathologic and behavioral deficits associated with CTE.

One of the most significant and characteristic pathologic changes is the accumulation of tau neurofibrillary tangles at the glia limitans and perivascular spaces. ${ }^{5}$ Puvenna and colleagues recently showed that glymphatic clearance of tau is impaired following repetitive traumatic brain injury in CTE specimens. ${ }^{6}$ We expanded upon these findings showing that TBI can contribute to tauopathy leading to CTE, Alzheimer's disease (AD), or, in rare cases, CTE/ AD. ${ }^{7}$ Hyperphosphorylated tau aggregates around venules and begins to form oligomers and neurofibrillary tangles. We recently showed that this perivascular tau was associated with an increase in endoplasmic reticulum (ER) stress activation. ${ }^{8}$ Additionally, we found that nicotinamide adenine dinucleotide phosphate (NADPH) oxidative stress was increased in neuronal membranes surrounding areas of acute BBB disruption. ${ }^{9}$

In this article, we show evidence of how secondary injury produces a CTE phenotype with our clinically relevant and validated Sprague-Dawley blast model. ${ }^{10}$ Blast exposure caused early activation of ER stress in response to acute BBB disruption. The opening of the $\mathrm{BBB}$ that we have previously reported occurs early

\footnotetext{
${ }^{1}$ Department of Neurosurgery, West Virginia University School of Medicine, Morgantown, West Virginia.

${ }^{2}$ Department of Basic Pharmaceutical Sciences, West Virginia University School of Pharmacy, Morgantown, West Virginia.

*The first two authors contributed equally.
} 
post-injury. ${ }^{11}$ This disruption allows further toxic particles, such as iron, to enter the brain and cause additional damage. Iron enters the brain from damaged red blood cells and heme degradation induced by blast exposure. Nisenbaum and colleagues have reported that iron-mediated toxicity can contribute to tau hyperphosphorylation and additional biochemical changes such as the formation of reactive oxygen species. ${ }^{12}$ ER stress weakens the cell, allowing iron to accumulate within the cytoplasm and damage the plasma membrane. We found that iron-mediated toxicity contributed to both tau hyperphosphorylation and conformational changes with our model. Salubrinal (Sal), an ER stress modulator, significantly reduced the iron-mediated toxicity.

These biochemical changes were linked to depressive and impulsive behavior measured with Forced Swim Test (FST) and Elevated Plus Maze (EPM), respectively. The tau changes were also associated with worsened cognitive performance measured with the Morris Water Maze (MWM). Importantly, we present data showing that ER stress modulation with Sal reduces acute injury cascades and improves behavior on the EPM. Using an in vitro assay, we found that Sal effectively reduced the neuroinflammatory marker nuclear factor kappa $\mathrm{B}(\mathrm{NF} \kappa \mathrm{B})$. This is in agreement with our recent article showing important "cross talk" between ER stress and neuroinflammatory cascades. ${ }^{8}$ Further work will mechanistically verify how ER stress modulation functions to improve behavior.

\section{Methods}

\section{Model and drug}

The Sprague-Dawley tabletop blast model produces a peak impulse of 50 psi. Finite element modeling reveals that the wave produced is $1 \mathrm{~ms}$ in duration (Fig. 1). The blast was delivered to the right side of the rat's skull with the head freely mobile and body protected by PVC tubing. The West Virginia University Animal Care and Use Committee approved this study, and all experiments were conducted in accordance with the national guidelines for the use and care of laboratory animals. A total of 120 3-6-month-old male Sprague- Dawley rats were used for this study. Sal (Tocris) was dissolved in $0.9 \%$ saline and administered at a concentration of $1 \mathrm{mg} / \mathrm{kg}$ at a single time 5 minutes post-blast. $0.9 \%$ saline was used for the vehicle in rats not receiving Sal.

\section{Study design}

Rats for the biochemical characterization studies were divided into control or blast groups based on time of euthanasia (30 min, $24 \mathrm{~h}$, or 21 days). The time points were chosen to look at 1) acute ER stress activation, 2) subsequent iron toxicity, and 3) tauopathy. Sham rats were anesthetized but not exposed to blast. The $n$ numbers for each assay are outlined in detail subsequently. The rats for behavior were divided according to assay and time point $(72 \mathrm{~h}$, 7 days, and 1 month).

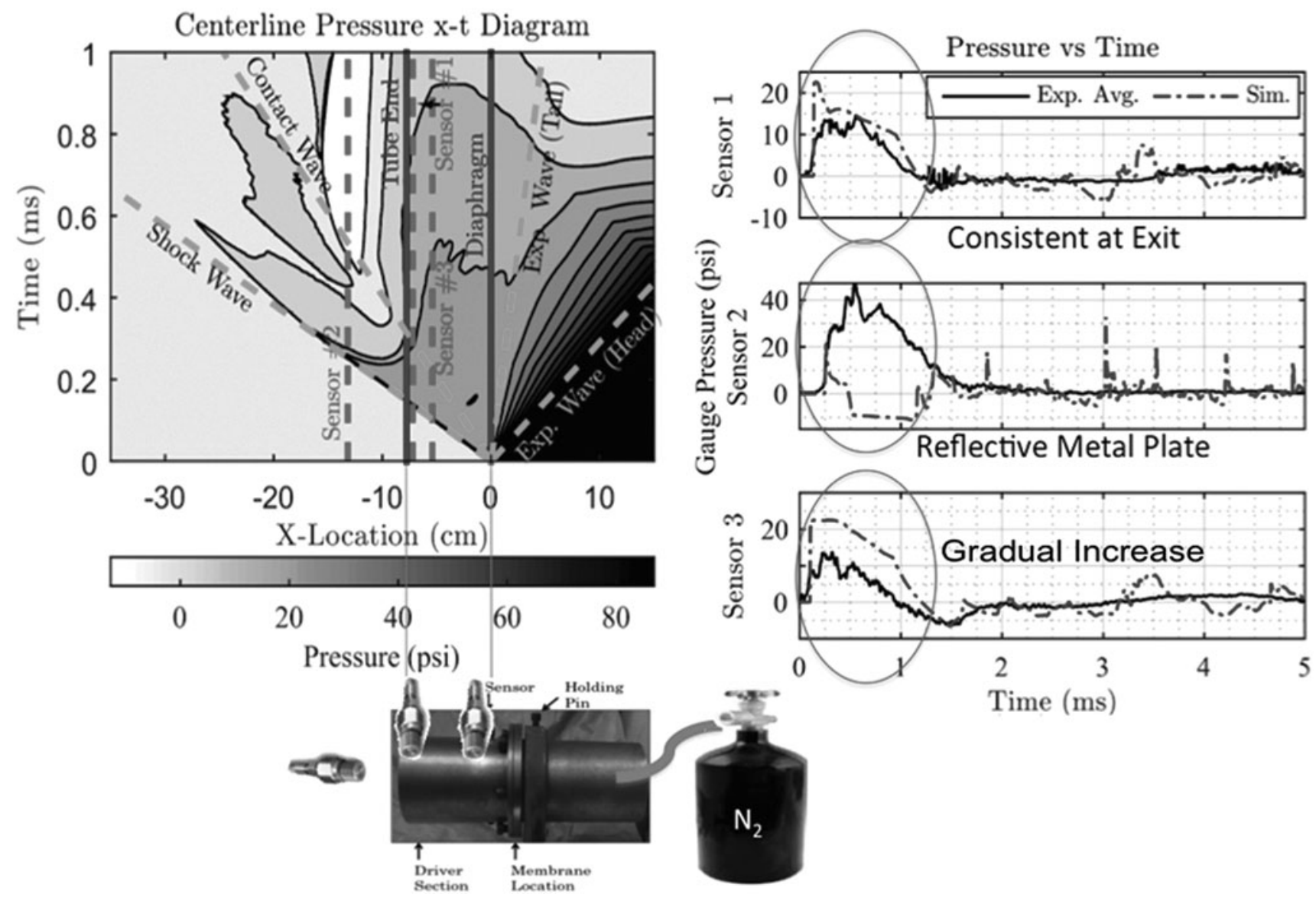

FIG. 1. Blast model schematic and finite element modeling representations showing that the tabletop model produces short duration waves, a rapid spike in pressure, and consistency in pressure readings at the tube exit. The shockwave combines with the contact wave and expansion wave to form a blast wave over time. 


\section{Cell culture}

NG-108 cells were cultured in a humidified incubator (5\% $\mathrm{CO}_{2}$ at $\left.37^{\circ} \mathrm{C}\right)$ in $\mathrm{T} 75$ culture flasks. High-glucose $(4.5 \mathrm{~g} / \mathrm{L})$ Dulbecco's Modified Eagle Medium (DMEM) was supplemented with $10 \%$ fetal bovine serum (FBS), penicillin, streptomycin, and hypoxanthine-aminopterin-thymidine (HAT) medium. When cells were $70 \%$ confluent, the media was replaced with differentiation media (DMEM with 5\% FBS, penicillin, streptomycin, HAT, and $0.5 \%$ dimethyl sulfoxide [DMSO]) and allowed to differentiate for an additional 3 days.

Differentiated cells were seeded $\left(1 \times 10^{5}\right)$ onto 12 well plates for treatment $(n=5)$. On each plate, wells were divided into four groups: 1) control, 2) Sal, 3) tunicamycin (TUN) + DMSO, and (4) TUN + SAL. TUN was administered $(1 \mu \mathrm{g} / \mathrm{mL})$ to cells for $6 \mathrm{~h}$ prior to collection to activate the ER stress response, and Sal was administered $(100 \mu \mathrm{M})$ to cells 30 min prior to collection. Control and TUN cells were administered DMSO $(0.5 \%) 30$ min prior to collection. Cells were collected in $1 \%$ sodium dodecyl sulfate (SDS) for measurement of protein expression.

\section{Western blot analysis}

For Western blot, frontal cortex was collected from blast and control rats, which has been shown previously to correlate with behavioral findings. ${ }^{13}$ In vitro cell collection was also conducted as stated. Protein samples were prepared in $1 \%$ SDS, and the assay was performed as previously described. ${ }^{14}$ Primary antibodies were rabbit anti-phosphoPERK $\mathrm{mAB}$ (1:1000), anti-PERK $\mathrm{mAB}$ (1:1000), and anti-phosphoeiF2 $\alpha$ (1:1000) (Cell Signaling); mouse anti-NF $\kappa$ B p65 (1:200) (Santa Cruz); mouse anti-AT8 (Thermo), and mouse anti-CP13 (kindly gifted by Peter Davies). A rabbit anti$\beta$-actin mAB $(1: 10,000)$ (Cell Signaling) was used as an endogenous control to normalize protein loading. Secondary antibodies were IRDye ${ }^{\circledR} 800 \mathrm{CW}$ (goat anti-rabbit) and IRDye ${ }^{\circledR}$ 680RD (goat anti-mouse) (LI-COR Biosciences). Images were collected and analyzed with an Odyssey fluorescent scanner. Images were converted to gray scale and the values calculated after background subtraction and then normalized to $\beta$-actin to measure relative intensity.

\section{Immunohistochemistry}

Brains were rapidly removed and placed into an ice-cold protease/phosphatase inhibitor cocktail mix (Halt ${ }^{\mathrm{TM}}$; Thermo Scientific; Pittsburgh, PA). Tissues were flash frozen in liquid nitrogen for storage at $-80^{\circ} \mathrm{C}$. Fixed brain tissue for the TBI and control rats were prepared as previously described. ${ }^{14}$ Briefly, rats were anesthetized with $4 \%$ isoflurane and cardiac perfused with ice-cold $0.9 \%$ saline, followed by $4 \%$ paraformaldehyde for $15 \mathrm{~min}$. Following perfusion, brains were removed and placed in $4 \%$ paraformaldehyde for $24 \mathrm{~h}$. Following fixation, the frontal cortex was sectioned into $4 \mathrm{~mm}$ slabs, which were then processed and embedded in paraffin, as previously described. ${ }^{15}$ Brain slabs were sectioned $(10 \mu \mathrm{m})$, mounted onto slides, and prepared for staining. Standard protocols for Perls DAB and cresyl violet were used for the iron staining (Sigma Aldrich).

\section{FST}

Depressive-like behavior was assessed with the FST assay. Two sets of rats were assessed: control and $72 \mathrm{~h}$ post-blast $(n=6$ per group). The apparatus was filled with water so that the rat could not touch the floor. On habituation day, the rat was placed in the apparatus for $15 \mathrm{~min}$ and monitored continuously. It was dried and warmed on a heating blanket following the trial. On test day, the rat was placed in the apparatus for $5 \mathrm{~min}$ and tracked with Any-Maze Video Tracking software (Stoelting Co.). Time spent immobile and time to first immobility episode was analyzed.

\section{EPM}

Impulsive-like behavior was investigated with the EPM assay. Four sets of rats were assessed: control, Sal, 7 day blast, and 7 day Sal + blast ( $n=9$ per group). The two arms of the EPM were $50 \times 10 \mathrm{~cm}$ and raised $60 \mathrm{~cm}$ from the floor. The closed arms had black siding $30 \mathrm{~cm}$ tall. The rat was placed in the middle of the EPM and tracking was performed with Any-Maze software for 5 min. The percentage time spent in the open arms and distance traveled was recorded and quantified.

\section{MWM}

Cognitive performance was evaluated with the MWM assay. Three sets of rats were assessed: control, 7 days post-blast, and 21 days post-blast ( $n=10$ per group). The circular pool was $180 \mathrm{~cm}$ in diameter. In the pool, a $10 \times 10 \mathrm{~cm}$ platform was submerged $2.5 \mathrm{~cm}$ below the surface of the water $\left(20^{\circ} \mathrm{C}\right)$. The training paradigm consisted of 6 days of acquisition with a hidden platform followed by a probe trial on day 7. During spatial acquisition, rats were placed into the maze apparatus four times from four different locations. Each rat had a $2 \mathrm{~min}$ trial to locate the platform. Timing stopped if the platform was found, but if the rat did not find it during the $2 \mathrm{~min}$ session, it was placed on the platform for $15 \mathrm{sec}$. On probe day, the rat was placed in the apparatus from a novel entrance point for $1 \mathrm{~min}$. An area encompassing 300\% of where the platform had been was outlined to indicate time spent in the appropriate region. Any-Maze was used to measure the distance traveled and the latency to reach the platform on acquisition days. On the probe trial day, it was used to measure how long the rat spent in the area $300 \%$ of where the platform had been.

\section{Statistical analysis}

Graphpad Prism software 5.0 (Graphpad Software, Inc.) was used for statistical analysis. A $t$ test, one way ANOVA, or two way ANOVA was used depending on the assay. $\chi^{2}$ analysis was used to quantify the iron staining. Tukey's post-hoc comparison was used for the one way and two way ANOVA. A $p$ value of $<0.05$ was considered statistically significant.

\section{Results}

\section{Phosphorylation states of PERK and elF2 $\alpha$ are elevated after bTBI}

To confirm ER stress activation following bTBI, two upstream components of the PERK-mediated ER stress pathway, PERK and eIF $2 \alpha$, were investigated for their respective phosphorylation levels. Western blot analyses showed a significant increase in PERK phosphorylation at $0.5 \mathrm{~h}$ following blast in the left prefrontal cortex (PFC) $(t=8.59 ; p<0.01)$ (Fig. 2A). At $0.5 \mathrm{~h}$ following blast exposure, a significant increase in eIF $2 \alpha$ phosphorylation was also found in the left PFC $(t=5.45 ; p<0.05)$ (Fig. 2B). No differences were seen in total PERK or total eIF2 $\alpha$ expression from the same samples (Fig. 2C,D), indicating a difference in phosphorylation state, but not in total protein expression (Fig. 2E,F). PERK and eIF2 $\alpha$ phosphorylation levels returned to baseline after $6 \mathrm{~h}$ post-blast (data not shown). Results showed a single moderate blast exposure to activate upstream constituents of the PERK-mediated ER stress pathway.

\section{Iron-mediated toxicity is increased following bTBI but is ameliorated by Sal administration}

It has previously been reported that non-heme iron accumulates in the brain after injury and contributes to robust cell loss. ${ }^{16}$ The 
A

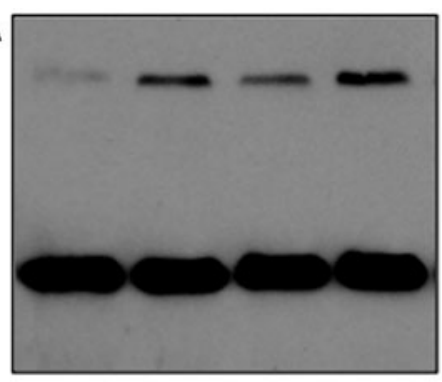

\section{P-PERK}

C $\quad$ B $\quad$ C $\quad$ B

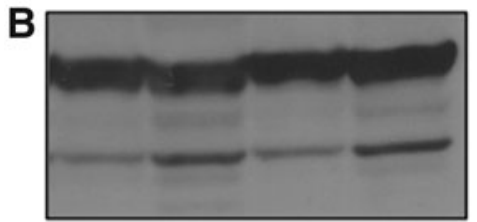

$\beta$-Actin

P-elF2 $\alpha$

C $\quad$ B $\quad$ C $\quad$ B

$\beta$-Actin

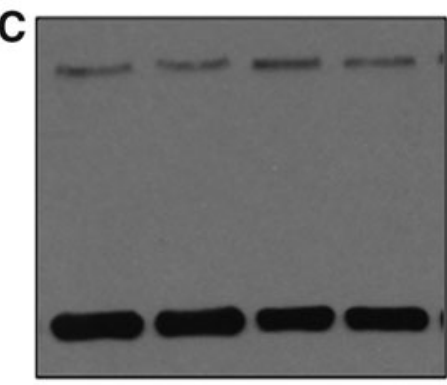

$\beta$-Actin

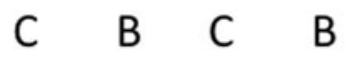

\section{Total PERK}

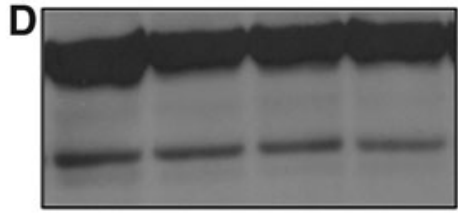

$\beta$-Actin

Total elF2 $\alpha$
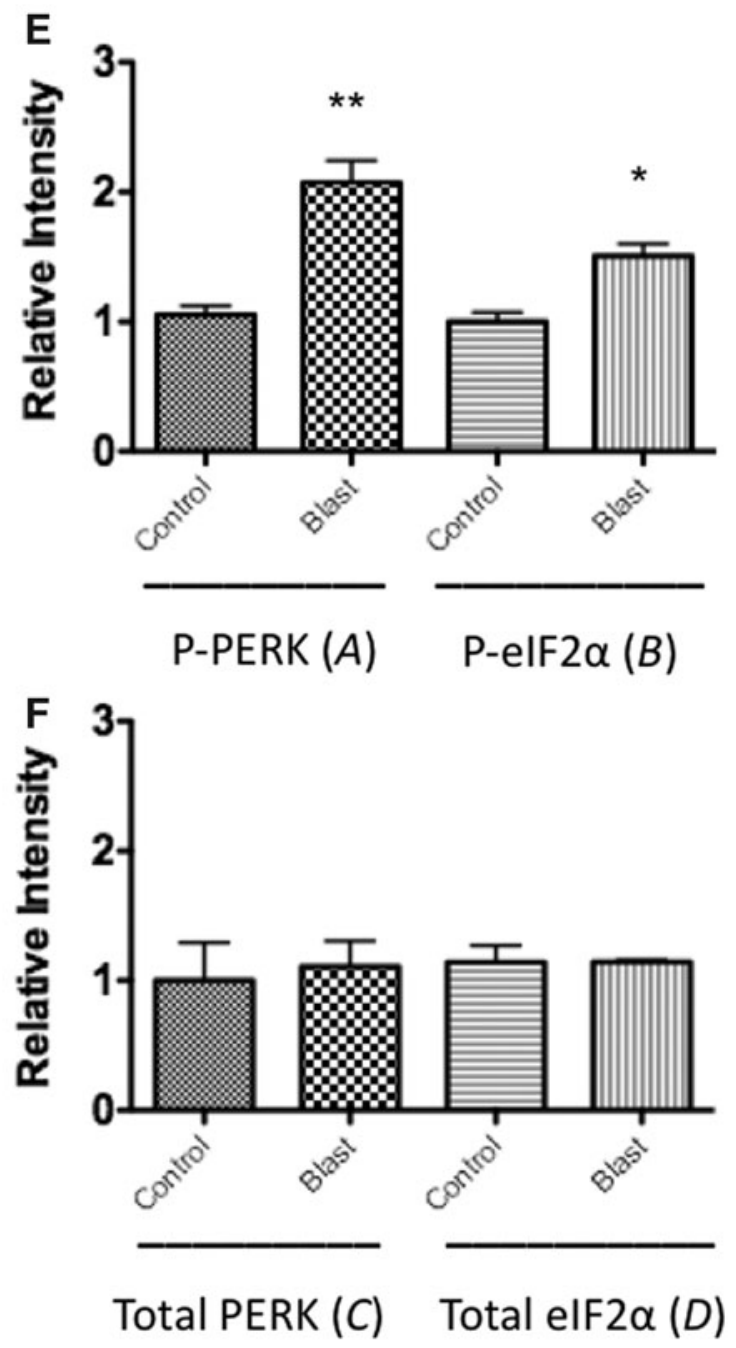

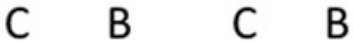

FIG. 2. Western blot analysis shows an increase in PERK and eIF2 $\alpha$ phosphorylation following blast exposure at acute time points. (A) Shows an increase in PERK phosphorylation at $30 \mathrm{~min}$ following blast exposure when compared with control animals $(t=8.591$; $p<0.01$ ). (B) Shows an increase in eIF $2 \alpha$ phosphorylation at $30 \mathrm{~min}$ following blast exposure when compared with control animals $(t=5.447 ; p<0.05)$. (C) Shows no increase in the expression of total PERK following blast exposure. (D) Shows no increase in the expression of total eIF $2 \alpha$ following blast exposure. ${ }^{*} p<0.01,{ }^{*} p<0.05$. (E, F) Representative graphs showing relative differences.

mechanisms by which this occurs are not fully known. In Figure 3 we show robust iron accumulation within neurons of the frontal cortex at $24 \mathrm{~h}$ post-TBI. A $\chi^{2}$ test revealed that severe TBI (sTBI) significantly increased the ratio of iron-positive stained cells $\left(\right.$ Control $=4 / 104$, Blast $=63 / 103$, and Blast + Sal $=24 / 108 ; \chi^{2}=59.46$; $p<0.001)$. Not surprisingly, ER stress modulation with Sal significantly prevented an increase in iron-positive cells, showing enhanced neuroprotective properties.

\section{Sal attenuated $N F_{\kappa} B$ administration} after in vitro TUN administration

TUN is a potent inducer of ER stress. We recently reported that ER stress activation contributes to robust neuroinflammation in vivo mediated by NFkB. ${ }^{16}$ What was unknown, was how ER stress modification altered the neuroinflammatory cascade. The activation of the neuroinflammatory cascade may be the 

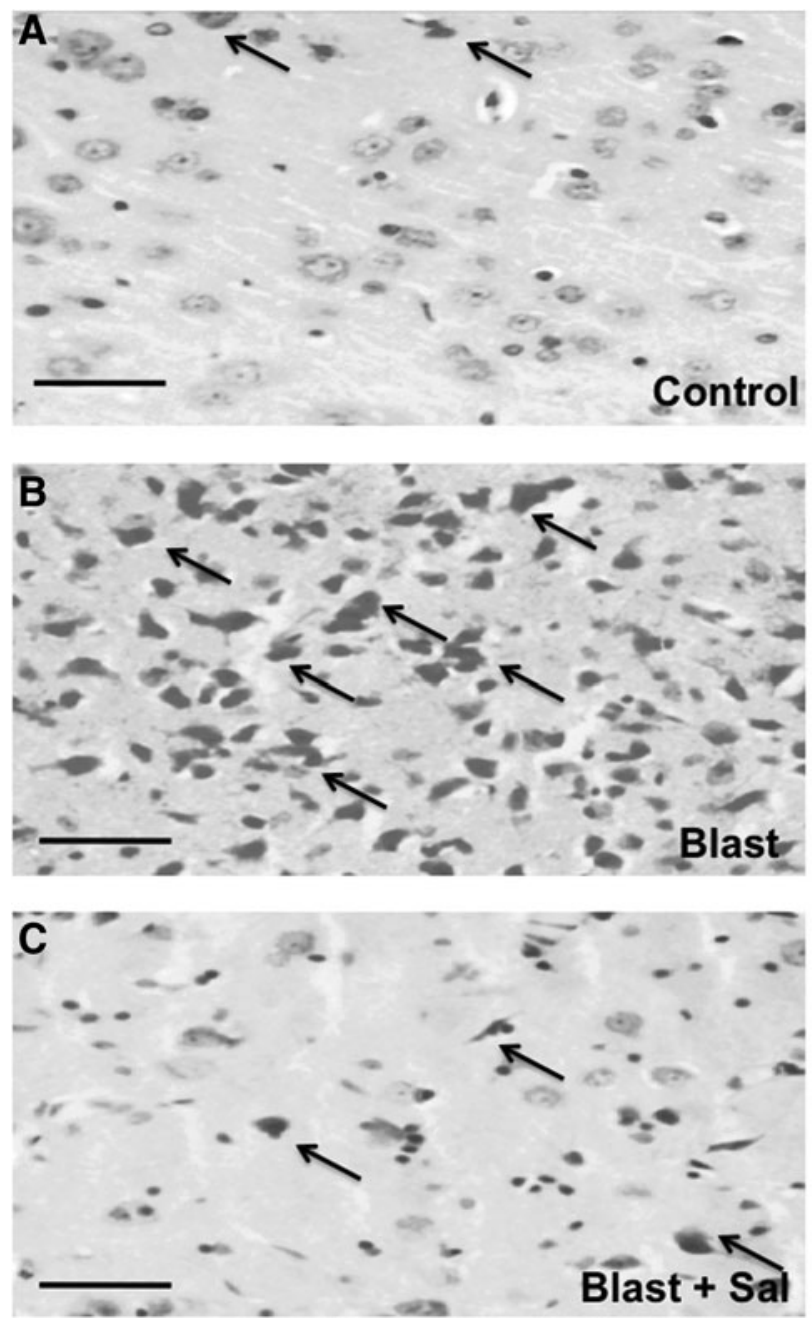

4/104 Cells Positive for Iron

\section{3/103 Cells Positive for Iron}

\section{4/108 Cells Positive for Iron}

FIG. 3. Iron staining in the frontal cortex shows a significant increase following blast traumatic brain injury (bTBI). Scale bar $=50 \mu \mathrm{m}$. (A) Iron accumulation in vehicle control is minimal (4/104 cells). (B) Iron accumulation following bTBI is significant (63/103 cells). (C) Iron accumulation following bTBI is prevented by the administration of salubrinal (Sal) $\left(24 / 108\right.$ cells). $\chi^{2}=59.46 ; p<0.001$. Arrows indicate representative cells where iron accumulation is pronounced in the cytoplasm.

contributing factor leading to iron toxicity. Figure 4 shows a significant difference in NFKB activity between groups of NG108 cells $\left(F_{(3,16)}=4.987 ; p<0.05\right)$. TUN + DMSO produced a significant increase in NFKB activity compared with control cells treated with DMSO $(q=4.766 ; p<0.05)$ or SAL $(q=4.432 ; p<0.05)$. TUN + Sal significantly attenuated NFKB activity compared with TUN + DMSO cells $(q=4.101 ; p<0.05)$. No difference between DMSO + SAL and TUN + SAL was observed. Future studies will examine this potential mechanism in relation to iron-mediated toxicity in vivo.

\section{bTBI increases depressive-like behavior}

A prominent symptom after blast TBI is the onset of depressive behavior. Recently, Tucker and colleagues reported depressive-like behavior in mice following bTBI. ${ }^{18}$ We show a similar phenotype of depressive-like behavior in our rat blast model: $72 \mathrm{~h}$ after blast, a significant difference between blast and control groups was seen for time spent immobile $(t=2.49 ; p<0.05)$. Blast rats spent $136.2 \pm$ $35.84 \mathrm{sec}$ immobile, whereas control rats spent $39.90 \pm 14.45 \mathrm{sec}$ immobile (Fig. 5A). No significant difference was seen for total immobility episodes $(t=1.2 ; p=0.26)$ (Fig. 5B). A significant difference was seen for time to first immobility session $(t=2.24$; $p<0.05)$. Blast-exposed rats had an average time of $9.35 \pm 6.16 \mathrm{sec}$ to first immobility episode, whereas control rats had an average time of $73.52 \pm 28.03 \mathrm{sec}$ to first immobility episode.

\section{Impulsive-like behavior is prevented by Sal administration}

A prominent symptom following blast exposure is the development of impulsivity. ${ }^{19}$ We have previously shown that the EPM is useful in determining impulsive-like behavior in rats. ${ }^{17}$ In this article, we found a significant difference between groups for time spent in the open arm $F_{(2,24)}=4.715 ; p<0.05$ (Fig. 6). Multiple comparisons revealed a significant difference between blast and control groups $(q=4.09 ; p<0.05)$. Blast-exposed rats spent on average $24.52 \mathrm{sec}$ in the open arm, whereas control rats spent $3.244 \mathrm{sec}$ in the open arm. Sal ameliorated the increase seen with blast $(q=3.31 ; p<0.05)$. Sal-treated rats spent on average only $7.3 \mathrm{sec}$ in the open arm.

\section{Blast exposure increases tau hyperphosphorylation and conformational change}

Tau pathology is a characteristic change showing evidence of neurodegeneration following neurotrauma. ${ }^{20}$ Tau can undergo 

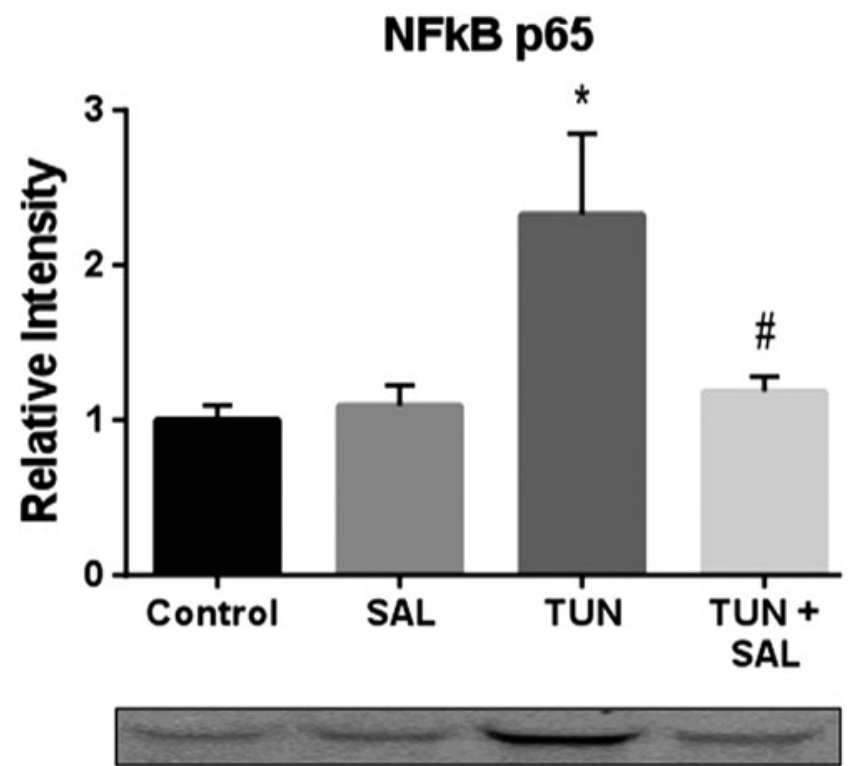

FIG. 4. Salubrinal (Sal) significantly reduced the activation of nuclear factor kappa $\mathrm{B}(\mathrm{NF} \kappa \mathrm{B})$ in vitro following endoplasmic reticulum (ER) stress induction with tunicamycin (TUN). A significant difference between TUN and control was seen for $\mathrm{NF} \kappa \mathrm{B}$ activation in NG-108 cells following treatment $(q=4.432$; $p<0.05)$. Sal significantly reduced the increase by TUN $(t=4.101$; $p<0.05) .{ }^{*} p<0.05$ for Control versus TUN groups. $\# p<0.05$ for TUN versus TUN + Sal groups.

biochemical modifications such as nitration and hyperphosphorylation. These modifications can lead to conformational changes in tau that predispose to pathologic aggregation. ${ }^{21}$ We observed a significant difference in tau changes between control and blast-exposed rats at 3 weeks post-injury. AT8, a marker of tau hyperphosphorylation, was significantly increased following blast $(t=3.59 ; p<0.05)$. $\mathrm{CP} 13$, a marker of tau comformational change, was also significantly increased following blast $(t=3.25 ; p<0.05)$ (Fig. 7).

\section{Cognitive deficits are increased following blast exposure}

bTBI can increase learning deficits and accelerate degenerative changes. ${ }^{22}$ MWM is a sensitive assay to detect these changes. We

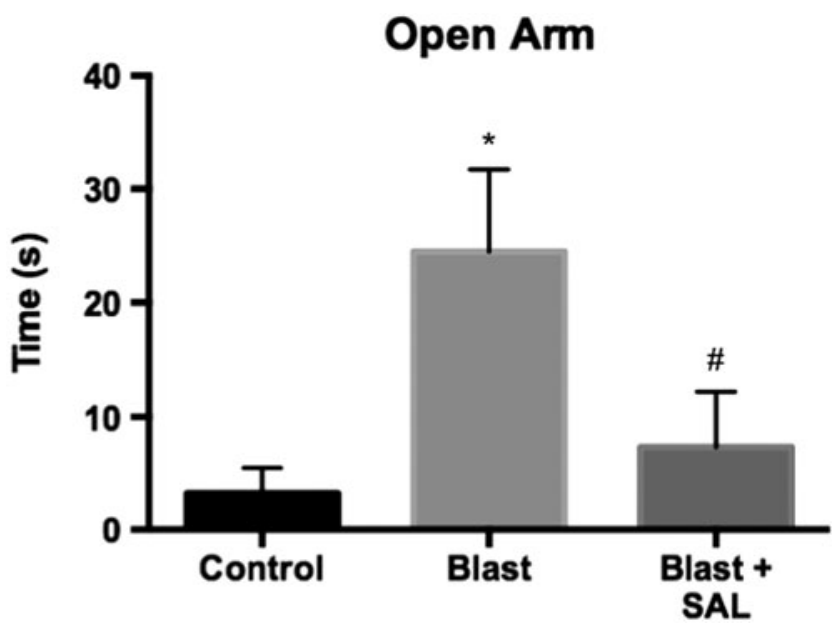

FIG. 6. Salubrinal (Sal) administration prevented the development of impulsive-like behavior following blast exposure $(n=9$ per group). A significant difference was seen in time spent in the open arm of the Elevated Plus Maze (EPM) between the control and blast groups $(q=4.09 ; p<0.05)$. Sal administration successfully prevented this increase in exploratory behavior in the open arm $(q=3.31 ; p<0.05) .{ }^{*} p<0.05$ for Control versus Blast groups. $\# p<0.05$ for Blast versus Blast + Sal groups.

found a significant difference between groups in escape latency $\left(F_{(2,19)}=18.58 ; p<0.001\right)$. At 3 weeks, blast-exposed rats had diminished performance on acquisition trials compared with control rats. Interestingly, a separate cohort of blast-exposed rats had no significant differences from controls when measured at 1 week post-injury. On the probe trial, a significant difference between groups was observed $\left(F_{(2,7)}=13.61 ; p<0.001\right)$. Rats exposed to blast and aged for 3 weeks spent less time in the area where the platform had been compared with controls $(q=6.51 ; \mathrm{P}<0.01)$ and with blast-exposed rats aged for 1 week $(q=6.44 ; p<0.01)$. No significant difference in swim speed was seen between groups $\left(F_{(2,7)}=0.209 ; p>0.05\right)$ (Fig. 8).

\section{Discussion}

Blast TBI can cause extensive changes to the brain that can lead to behavioral deficits such as executive dysfunction, memory deficits, and cognitive impairments in war fighters. ${ }^{23}$ The underlying pathophysiology behind these behavior changes is poorly understood. In this article, we found that rats exposed to blast had
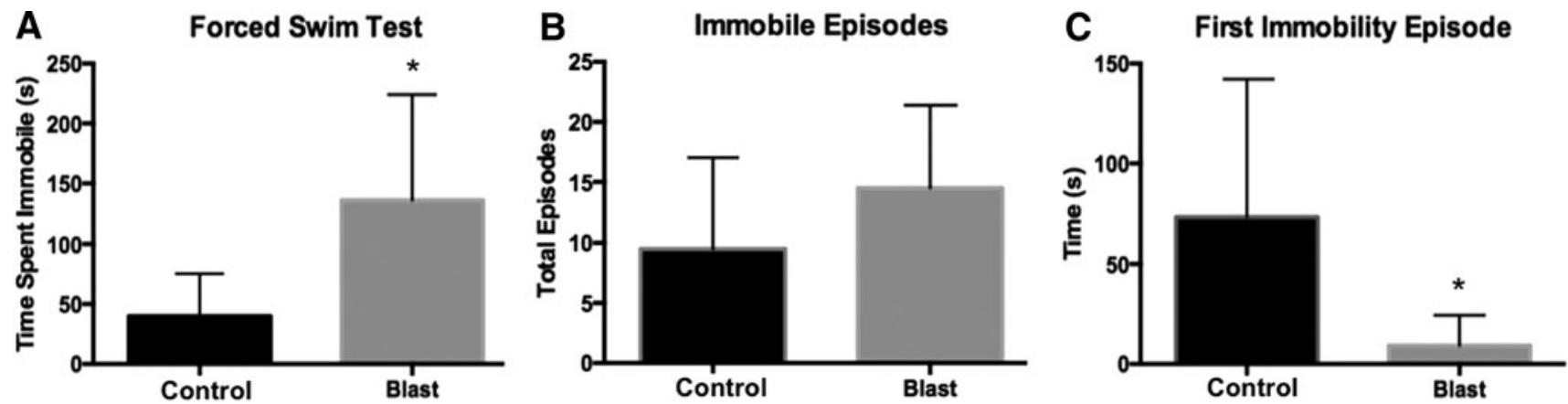

FIG. 5. Blast exposure significantly increased depressive-like behavior ( $n=6$ per group). (A) Rats exposed to blast spent more time immobile compared with control rats $(t=2.49, p<0.05)$. (B) No significant difference was seen on total number of immobility episodes. (C) The latency to first immobility episode was significantly shortened for the blast-exposed rats $(t=2.24, p<0.05)$. ${ }^{*} p<0.05$. 
A

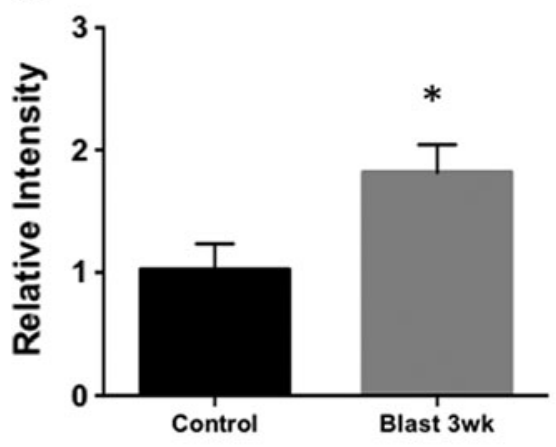

AT8

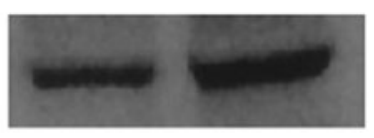

Actin

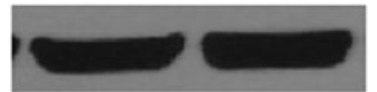

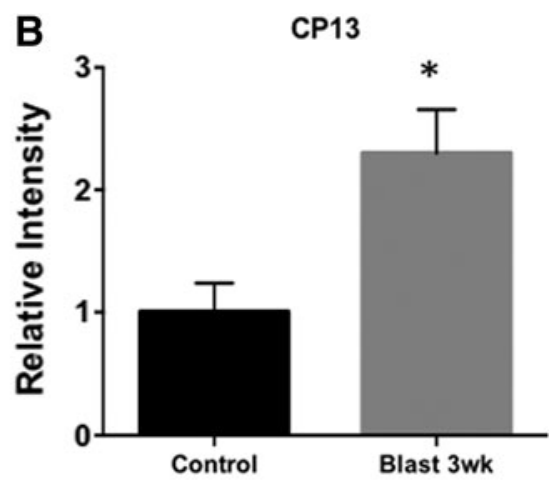

CP13

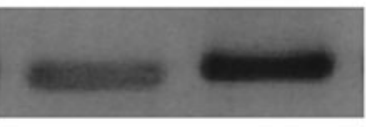

Actin

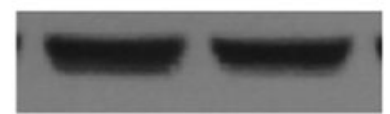

FIG. 7. Blast exposure increases tauopathy changes. (A) AT8, a marker of tau hyperphosphorylation, was increased in the contralateral cortex 3 weeks after blast exposure $(t=3.59 ; p<0.05)$. (B) CP13, a marker of tau conformational change, was increased in the cortex as well 3 weeks after blast exposure $(t=3.25 ; p<0.05)$. ${ }^{*} p<0.05$.

increased depressive-like and impulsive-like behavior and cognitive deficits. Interestingly, the timing of these changes was sequential. Rats displayed depressive-like symptoms at $72 \mathrm{~h}$ postinjury and impulsive-like behavior at 7 days. A separate cohort of rats with the same blast conditions was used for the MWM testing. Surprisingly, the 7 day blast group showed no changes in cognitive performance whereas the 3 week blast group did. These changes are in agreement with the progressive symptom development often reported in patients with CTE. ${ }^{24}$ The more rapid progression seen in the rodent model is likely the result of species differences and the shortened life span of the animals compared with humans.

To investigate the underlying pathophysiology linking neurotrauma to neurodegenerative disease, we looked at the activation of the ER stress response as well as iron-mediated toxicity. Chen and colleagues proposed that iron accumulation released from degrading red blood cells contributes to robust inflammatory and secondary injury responses in the brain. ${ }^{25} \mathrm{We}$ found that ER stress contributed to the activation of iron toxicity, but that Sal prevented this activation. In our previous article, we found an association among ER stress, oxidative stress, and neuroinflammation. ${ }^{26}$ Using in vitro investigation, we verified that reducing TUN mediated ER stress significantly, mitigated the activation of $\mathrm{NF} \kappa \mathrm{B}$. The reduction in neuroinflammation mediated by ER stress modulation may have beneficial effects on behavior. Faden and colleagues have shown that persistent neuroinflammation is the key player in the development of chronic neurodegeneration. ${ }^{27}$

Preliminary evidence showed that when we administered Sal post-blast, impulsive-like behavior was prevented. As the injury progressed with time, tau changes began to occur, and induced impairment in learning as measured with the MWM. Gerson and colleagues likewise showed that tau oligomer formation following blast exposure could contribute to cognitive impairment. ${ }^{21}$ Going forward, we want to investigate the progression of tauopathy at later time points. This will include an assessment of both oligomer formation and cis/trans tau configuration similar to that being performed by Kanaan and colleagues in human CTE tissue. ${ }^{28} \mathrm{We}$ propose that as the rats age over a series of months, they will develop progressive pathologic tau oligomers.

Most importantly, we will investigate whether ER stress modulation can prevent long-term behavior. We have shown in our
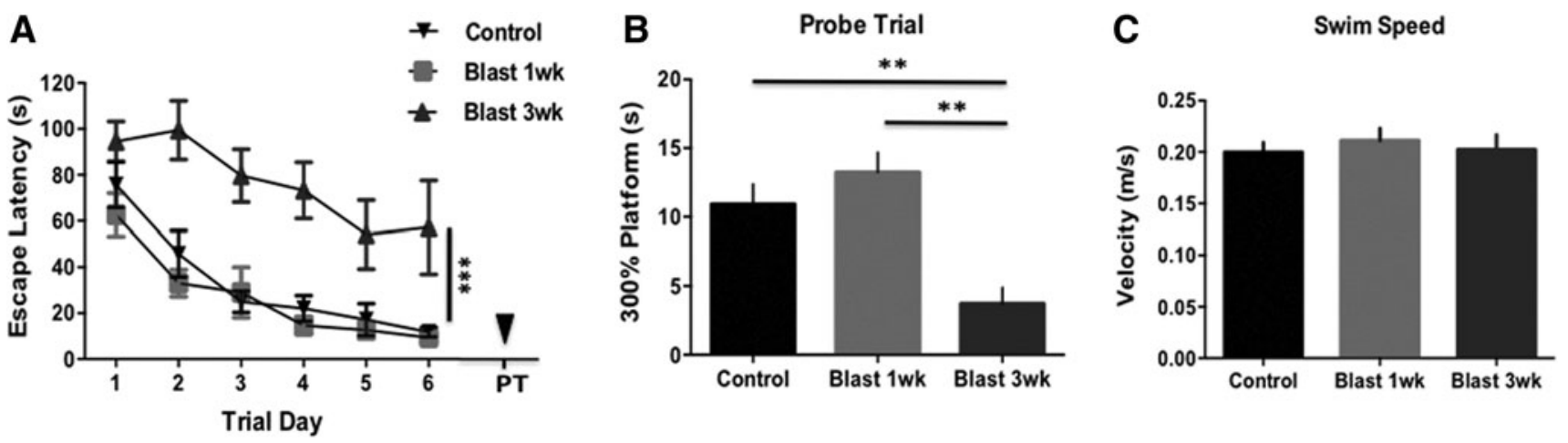

FIG. 8. Blast exposure causes learning impairment at 3 weeks post-injury but not as early as 7 days $(n=10$ per group). A significant difference was seen between groups on $(\mathbf{A})$ acquisition trials $\left(F_{(2,19)}=18.58 ; p<0.001\right)$ and $(\mathbf{B})$ the probe trial $\left(F_{(2,7)}=13.61 ; p<0.001\right)$. No significant difference was seen in (C) swim speed. $* * * p<0.001, * * p<0.01$. 
recent Journal of Neurosurgery and Frontiers in Neuroscience articles that reducing ER stress is protective at subacute time points. ${ }^{8,13}$ A multi-drug regimen targeting different secondary injury cascades at different sequential time points will likely have the most efficacy. We have previously shown benefit in targeting the NADPH oxidative stress response, which can preserve plasma membranes and prevent iron damage. ${ }^{9}$ Iron damage is further increased post-blast disruption. Targeting BBB disruption and oxidative stress may, therefore, be additional strategies to be used in conjunction with ER stress modulation.

\section{Conclusion}

In conclusion, ER stress activation contributes to iron damage. In vitro investigation confirmed that $\mathrm{ER}$ stress increases $\mathrm{NF} \kappa \mathrm{B}$ activation, but that this can be prevented by the administration of Sal. Sal reduced exploratory behavior on the EPM. Going forward, we will investigate how tauopathy progresses and contributes to long-term behavior changes. Once this has been characterized, a multi-drug regimen will be established to prevent the onset and duration of neuroinflammation. We propose that this multi-drug regimen may prove beneficial in preventing neurodegeneration following neurotrauma.

\section{Acknowledgments}

Aric Logsdon and Brandon Lucke-Wold thank the American Foundation of Pharmaceutical Education for awarding pre-doctoral fellowships. Brandon Lucke-Wold also received funding from Sigma Xi Grants in Aid of Research, Neurosurgery Research and Education Foundation Medical Student Summer Fellowship, American Association of Pharmaceutical Scientists Pre-doctoral Fellowship, and the American Medical Association Foundation Seed Grant.

\section{Author Disclosure Statement}

No competing financial interests exist.

\section{References}

1. Doherty, C.P., O'Keefe, E., Wallace, E., Loftus, T., Keaney, J., Kealy, J., Humphries, M.M., Molloy, M.G., Meaney, J.F., Farrell, M., and Campbell, M. (2016). Blood-brain barrier dysfunction as a hallmark pathology in chronic traumatic encephalopathy. J. Neuropathol. Exp. Neurol. 75, 656-672.

2. Shively, S.B., Horkayne-Szakaly, I., Jones, R.V., Kelly, J.P., Armstrong, R.C., and Perl, D.P. (2016). Characterisation of interface astroglial scarring in the human brain after blast exposure: a postmortem case series. Lancet Neurol. 15, 944-953.

3. Stemper, B.D., Shah, A.S., Budde, M.D., Olsen, C.M., GlavaskiJoksimovic, A., Kurpad, S.N., McCrea, M., and Pintar, F.A. (2016). Behavioral outcomes differ between rotational acceleration and blast mechanisms of mild traumatic brain injury. Front. Neurol. 7, 31.

4. Kabu, S., Jaffer, H., Petro, M., Dudzinski, D., Stewart, D., Courtney, A., Courtney, M., and Labhasetwar, V. (2015). Blast-associated shock waves result in increased brain vascular leakage and elevated ROS levels in a rat model of traumatic brain injury. PLoS One 10, e0127971.

5. Pan, J., Connolly, I.D., Dangelmajer, S., Kintzing, J., Ho, A.L., and Grant, G. (2016). Sports-related brain injuries: connecting pathology to diagnosis. Neurosurg. Focus 40, E14.

6. Puvenna, V., Engeler, M., Banjara, M., Brennan, C., Schreiber, P., Dadas, A., Bahrami, A., Solanki, J., Bandyopadhyay, A., Morris, J.K., Bernick, C., Ghosh, C., Rapp, E., Bazarian, J.J., and Janigro, D. (2016). Is phosphorylated tau unique to chronic traumatic encephalopathy? Phosphorylated tau in epileptic brain and chronic traumatic encephalopathy. Brain Res. 1630, 225-240.
7. Turner, R.C., Lucke-Wold, B.P., Robson, M.J., Lee, J.M., and Bailes, J.E. (2016). Alzheimer's disease and chronic traumatic encephalopathy: Distinct but possibly overlapping disease entities. Brain Inj. 11, $1-14$.

8. Lucke-Wold, B.P., Turner, R.C., Logsdon, A.F., Nguyen, L., Bailes, J.E., Lee, J.M., Robson, M.J., Omalu, B.I., Huber, J.D. and Rosen, C.L. (2016). Endoplasmic reticulum stress implicated in chronic traumatic encephalopathy. J. Neurosurg. 124, 687-702.

9. Lucke-Wold, B.P., Naser, Z.J., Logsdon, A.F., Turner, R.C., Smith, K.E., Robson, M.J., Bailes, J.E., Lee, J.M., Rosen, C.L., and Huber, J.D. (2015). Amelioration of nicotinamide adenine dinucleotide phosphate-oxidase mediated stress reduces cell death after blastinduced traumatic brain injury. Transl. Res. 166, 509-528.

10. Turner, R.C., Naser, Z.J., Logsdon, A.F., DiPasquale, K.H., Jackson, G.J., Robson, M.J., Gettens, R.T., Matsumoto, R.R., Huber, J.D., and Rosen, C.L. (2013). Modeling clinically relevant blast parameters based on scaling principles produces functional \& histological deficits in rats. Exp. Neurol. 248, 520-529.

11. Lucke-Wold, B.P., Logsdon, A.F., Smith, K.E., Turner, R.C., Alkon, D.L., Tan, Z., Naser, Z.J., Knotts, C.M., Huber, J.D., and Rosen, C.L. (2015). Bryostatin-1 restores blood brain barrier integrity following blast-induced traumatic brain injury. Mol. Neurobiol. 52, 11191134.

12. Nisenbaum, E.J., Novikov, D.S., and Lui, Y.W. (2014). The presence and role of iron in mild traumatic brain injury: an imaging perspective. J. Neurotrauma 31, 301-307.

13. Logsdon, A.F., Turner, R.C., Lucke-Wold, B.P., Robson, M.J., Naser, Z.J., Smith, K.E., Matsumoto, R.R., Huber, J.D., and Rosen, C.L. (2014). Altering endoplasmic reticulum stress in a model of blastinduced traumatic brain injury controls cellular fate and ameliorates neuropsychiatric symptoms. Front. Cell Neurosci. 8, 421.

14. Lucke-Wold, B.P., Logsdon, A.F., Smith, K.E., Turner, R.C., Alkon, D.L., Tan, Z., Naser, Z.J., Knotts, C.M., Huber, J.D., and Rosen, C.L. (2014). Bryostatin-1 restores blood brain barrier integrity following blast-induced traumatic brain injury. Mol. Neurobiol.

15. Turner, R.C., Naser, Z.J., Bailes, J.E., Smith, D.W., Fisher, J.A. and Rosen, C.L. (2012). Effect of slosh mitigation on histologic markers of traumatic brain injury: laboratory investigation. J. Neurosurg. 117, $1110-1118$.

16. Chen-Roetling, J., Liu, W., and Regan, R.F. (2011). Iron accumulation and neurotoxicity in cortical cultures treated with holotransferrin. Free Radic. Biol. Med. 51, 1966-1974.

17. Logsdon, A.F., Lucke-Wold, B.P., Nguyen, L., Matsumoto, R.R., Turner, R.C., Rosen, C.L., and Huber, J.D. (2016). Salubrinal reduces oxidative stress, neuroinflammation and impulsive-like behavior in a rodent model of traumatic brain injury. Brain Res. 1643, $140-151$.

18. Tucker, L.B., Burke, J.F., Fu, A.H., and McCabe, J.T. (2016). Neuropsychiatric symptom modeling in male and female C57BL/6J mice after experimental traumatic brain injury. J Neurotrauma. [Epub ahead of print.]

19. Harch, P.G., Andrews, S.R., Fogarty, E.F., Amen, D., Pezzullo, J.C., Lucarini, J., Aubrey, C., Taylor, D.V., Staab, P.K., and Van Meter, K.W. (2012). A phase I study of low-pressure hyperbaric oxygen therapy for blast-induced post-concussion syndrome and posttraumatic stress disorder. J. Neurotrauma 29, 168-185.

20. Turner, R.C., Lucke-Wold, B.P., Logsdon, A.F., Robson, M.J., Dashnaw, M.L., Huang, J.H., Smith, K.E., Huber, J.D., Rosen, C.L., and Petraglia, A.L. (2015). The quest to model chronic traumatic encephalopathy: a multiple model and injury paradigm experience. Front. Neurol. 6, 222.

21. Gerson, J., Castillo-Carranza, D.L., Sengupta, U., Bodani, R., Prough, D.S., DeWitt, D.S., Hawkins, B.E., and Kayed, R. (2016). Tau oligomers derived from traumatic brain injury cause cognitive impairment and accelerate onset of pathology in Htau mice. J. Neurotrauma. 33, 2034-2043.

22. Tweedie, D., Rachmany, L., Rubovitch, V., Li, Y., Holloway, H.W., Lehrmann, E., Zhang, Y., Becker, K.G., Perez, E., Hoffer, B.J., Pick, C.G., and Greig, N.H. (2016). Blast traumatic brain injury-induced cognitive deficits are attenuated by preinjury or postinjury treatment with the glucagon-like peptide-1 receptor agonist, exendin-4. Alzheimer's Dement. 12, 34-48.

23. Daneshvar, D.H., Goldstein, L.E., Kiernan, P.T., Stein, T.D., and McKee, A.C. (2015). Post-traumatic neurodegeneration and chronic traumatic encephalopathy. Mol. Cell. Neurosci. 66, 81-90. 
24. Galgano, M.A., Cantu, R. and Chin, L.S. (2016). Chronic traumatic encephalopathy: the impact on athletes. Cureus 8, e532.

25. Chen, H., Constantini, S., and Chen, Y. (2015). A two-model approach to investigate the mechanisms underlying blast-induced traumatic brain injury. In: Brain Neurotrauma: Molecular, Neuropsychological, and Rehabilitation Aspects. F.H. Kobeissy (ed). Boca Raton, FL, Chapter 17.

26. Logsdon, A.F., Lucke-Wold, B.P., Nguyen, L., Matsumoto, R.R., Turner, R.C., Rosen, C.L. and Huber, J.D. (2016). Salubrinal reduces oxidative stress, neuroinflammation and impulsive-like behavior in a rodent model of traumatic brain injury. Brain Res. 1643, 140-151.

27. Faden, A.I., Wu, J., Stoica, B.A., and Loane, D.J. (2016). Progressive inflammation-mediated neurodegeneration after traumatic brain or spinal cord injury. Br. J. Pharmacol. 173, 681-691.

28. Kanaan, N.M., Cox, K., Alvarez, V.E., Stein, T.D., Poncil, S., and McKee, A.C. (2016). Characterization of early pathological tau con- formations and phosphorylation in chronic traumatic encephalopathy. J. Neuropathol. Exp. Neurol. 75, 19-34.

Address correspondence to:

Charles L. Rosen, MD, PhD

Department of Neurosurgery

West Virginia University School of Medicine

One Medical Center Drive

Suite 4300, Health Sciences Center

PO Box 9183

Morgantown, $W V$ 26506-9183

E-mail: crosen@hsc.wvu.edu 